\title{
QUALITATIVE PROPERTIES OF THE FREE-BOUNDARY OF THE REYNOLDS EQUATION IN LUBRICATION
}

\author{
S.J. AlVAREZ
}

Abstract

The hidrodynamic lubrication of a cylindrical bearing is governed by the Reynolds equation that must be satisfied by the preassure of lubricating oil. When cavitation occurs we are carried to an elliptic free-boundary problem whcre the free-boundary separates the lubricated region from the cavited region.

Some qualitative properties are obtained about the shape of the free-boundary as well as the localization of the cavited region.

\section{Introduction. Existence and uniqueness}

Let $\Omega$ be the rectangle $(0,2 \pi) \times(0,1) \subset \mathbf{R}^{2} ;$ let $\Gamma_{0}=(0,2 \pi) \times\{0\}, \Gamma_{1}=$ $(0,2 \Pi) \times\{1\}$ and let us introduce the following sets of functions:

$$
\begin{aligned}
& V=\left\{\phi \in H^{1}(\Omega),\left.\phi\right|_{\Gamma_{0} u \Gamma_{1}}=0, \phi \text { is } 2 \pi x-\text { periodic }\right\} \\
& V_{a}=\left\{\phi \in H^{1}(\Omega),\left.\phi\right|_{\Gamma_{0}}=0,\left.\phi\right|_{\Gamma_{1}}=p_{a}, \phi \text { is } 2 \pi x-\text { periodic }\right\}
\end{aligned}
$$

where $H^{1}(\Omega)$ is the Sobolev space of functions such that they and their first derivatives are square summable.

We consider the following:

Problem $(P)$.

Find a pair of functions $(p, \gamma)$ such that

(1.1) $(p, \gamma) \in V_{a} \times L^{\infty}(\Omega)$

(1.2) $p \geq 0$ and $H(p) \leq \gamma \leq 1$ a.e. in $\Omega$

(1.3) $\int_{\Omega} h^{3} \nabla p \nabla \xi=\int_{\Omega} h \gamma \frac{\partial \xi}{\partial x} \quad \forall \xi \in V$,

where $h=h(x)=1+\alpha \cos x$, with $0<\alpha<1$, and $H$ is the Heaviside function.

This problem is related to the lubrication with cavitation arising in bearings. The first unknow is the pressure distribution $-p-$ in a thin film of lubricant contained in the narrow gap between two circular cylinders of parallel axes (the shaft and the bearing); another unknow is the percentage $-\gamma-$ of oil contained in an elementary volume. 
shaft and the bearing); another unknow is the percentage $-\gamma-$ of oil contained in an elementary volume.

Introducing cylindrical coordinates, the gap $h$ depends only on the angular coordinate, being $\alpha$ the eccentricity ratio of the bearing.

The equation (1.3) derives from the Reynolds equation, $\operatorname{div}\left(h^{3} \nabla p\right)=h^{\prime}$, which must be satisfied for $p$ on the region $[p>0]$, and from conservation laws of flow across the free boundary separing the regions $[p>0]$ and $[p=0]$ in $\Omega$. In the lubricated region (completely occuped for oil) $\gamma$ is equal to one, while over the cavited region $([p=0]) \gamma$ must satisfy $0 \leq \gamma \leq 1$.

The main goal of this paper is to give some qualitative properties of the free-boundary,

$$
\Gamma=\overline{[p>0]} \cap \overline{[p=0]} \cap \Omega .
$$

The existence of solutions for Problem $(P)$ was proved by Bayada and Chambat in [B-Ch]; they prove also uniqueness of solutions under the assumption that the free-boundary is a Lipschitz-continuous function of $x$. A comparison result and uniqueness was proved by Carrillo and the author in [A-C], without any of the previous assumption related to the free-boundary.

For a more general treatment on physical aspects and the formulation of Problem $(P)$, see $[\mathbf{A}],[\mathrm{B}-\mathbf{C h}],[\mathbf{D}-\mathbf{T}],[\mathbf{F}]$.

About existence and uniqueness, we recall the following results:

Theorem 1.1. (Existence and Regularity)

There exist at least one solution for Problem $(P)$; moreover, if $(p, \gamma)$ satisfies (1.1), (1.2) and (1.9), then

$$
p \in C^{0}(\bar{\Omega}) \cap C^{0, r}(\Omega \cup(\{0\} \times(0,1)) \cup(\{2 \pi\} \times(0,1))) .
$$

Proof: See $[\mathrm{B}-\mathrm{Ch}]$ and $[\mathrm{A}-\mathrm{C}]$, as well as the proof of existence for the Dam Problem in [B-K-S].

\section{Theorem 1.2. (Comparison) ([A-C] )}

Let $\left(p_{1}, \gamma_{1}\right)$ and $\left(p_{2}, \gamma_{2}\right)$ be two pairs in $H^{1}(\Omega) \times L^{\infty}(\Omega)$, with $p_{1}$ and $p_{2}$ being $2 \Pi x$-periodic functions and satisfying (1.2) and (1.9), as well as the condition,

(1.4) $\left.p_{i}\right|_{\Gamma_{j}}=\phi_{i}^{j}$ for $i=1,2$ and $j=0,1$

where for $\phi_{i}^{j}$ we assume

(1.5) $\phi_{i}^{j} \in C\left(\Gamma_{j}\right)$ and $\phi_{1}^{j} \leq \phi_{2}^{j}$.

Then $p_{1} \leq p_{2}$ in $\Omega$.

Like a corollary of this theorem, we have:

Theorem 1.3. (Uniqueness) $([\mathrm{A}-\mathrm{C}])$ 
There exist an unique solution $(p, \gamma)$ for Problem $(P)$.

Remark. Theorem 1.2 gives a global comparison result in $\Omega$ for $p_{1}$ and $p_{2}$, when we can compare their values on $\Gamma_{0}$ and $\Gamma_{1}$ : this remain true to compare solutions of Problem $(P)$ with solutions of a swiftly modified problem, as we will precise later in Section 3.

\section{Uniforme bounds for solutions in the $x$-variable}

In this section we shall give an upper bound and a lower bound, both independents of $x$, for solutions of Problem $(P)$.

Let $M=\operatorname{maximum}_{x \in[0,2 \pi]} \frac{h^{\prime}(x)}{h^{3}(x)}$, and, for $0 \leq y \leq 1$, let us define,

$$
\begin{aligned}
& \bar{v}(y)=-\frac{M}{2} y^{2}+\left(p_{\alpha}-\frac{M}{2}\right) y \\
& \underline{v}(y)=\left[\frac{M}{2} y^{2}+\left(p_{\alpha}-\frac{M}{2}\right) y\right]^{+}
\end{aligned}
$$

Such functions satisfy:

$$
\begin{aligned}
& \underline{v}(0)=\bar{v}(0)=0 \\
& \underline{v}(1)=\bar{v}(1)=p_{a} \\
& \bar{v}^{\prime \prime}=-M \\
& \underline{v}^{\prime \prime}=\left\{\begin{array}{l}
0 \text { if } y<1-2 p_{a} / M \\
M \text { if } y>1-2 p_{a} / M
\end{array}\right.
\end{aligned}
$$

We have:

Theorem 2.1.

If $(p, \gamma)$ is the solution of Problem $(P)$, then

$$
p(x, y) \leq \bar{v}(y) \quad \text { in } \ddot{\Omega} .
$$

Proof: Taking $\xi=(p-\bar{v})^{+}$, and as $\gamma=1$ on the support of $\xi$, we have

$$
\int_{\Omega} h^{3} \nabla p \nabla \xi=\int_{\Omega} h \gamma \xi_{x}=-\int_{\Omega} h^{\prime} \xi
$$

Moreover,

$$
\int_{\Omega} h^{3} \nabla \bar{v} \nabla \xi=\int_{\Omega} h^{3} \bar{v}^{\prime} \xi_{y}=-\int_{\Omega} h^{3} \bar{v}^{\prime \prime} \xi=\int_{\Omega} h^{3} M \xi \geq \int_{\Omega} h^{\prime} \xi .
$$


and, substracting from the above equality:

$$
\int_{\Omega} h^{3}\left|\nabla(p-\bar{v})^{+}\right|^{2}=\int_{\Omega} h^{3} \nabla(p-\bar{v}) \nabla \xi \leq 0 .
$$

So, we obtain

$$
(p-\bar{v})^{+}=\text {constant } \quad \text { in } \Omega
$$

and, hence

$$
(p-\bar{v})^{+}=0 \quad \text { i.e. } p \leq \bar{v} .
$$

In order to complet the boundedness of $p$, we have:

\section{Theorem 2.2.}

If $(p, \gamma)$ is the solution of Problem $(P)$, then

$$
p(x, y) \geq \underline{v}(y) \quad \text { in } \bar{\Omega} .
$$

Proof: Let $\xi=(\underline{v}-p)^{+}$; we have:

$$
\int_{\Omega} h^{3} \nabla \underline{v} \nabla \xi=\int_{\Omega} h^{3} \underline{v}^{\prime} \xi_{y}=-\int_{\Omega} h^{3} \underline{v}^{\prime \prime} \xi=-\int_{\Omega} h^{3} M \xi \leq-\int_{\Omega} h^{\prime} \xi,
$$

since $\underline{v}^{\prime \prime}(y)=M$ if $\underline{v} \neq 0$, and hence on the support of $\xi$.

Now, since $\xi_{x}=\left[(\underline{v}-p)^{+}\right]_{x}=0$, on the region $[p=0]$, we have:

$$
\int_{\Omega} h^{3} \nabla p \nabla \xi=\int_{\Omega} h \gamma \xi_{x}=\int_{\Omega} h \xi_{x}+\int_{\Omega} h(\gamma-1) \xi_{x}=-\int_{\Omega} h^{\prime} \xi
$$

and so:

$$
\int_{\Omega} h^{3}\left|\nabla(\underline{v}-p)^{+}\right|^{2}=\int_{\Omega} h^{3} \nabla(\underline{v}-p) \nabla \xi \leq 0 .
$$

Similarly to Theorem 2.1 , we obtain the conclusion.

\section{Corollary 2.3.}

If $(p, \gamma)$ is the solution of Problem $(P)$, with $p_{a} \geq M / 2$, then $p>0$ in $\Omega$ and so there is not free-boundary.

Proof: If $p_{a} \geq M / 2$ then $\underline{v}(y)>0$ and $p>0$ for all $y \in(0,1)$. 


\section{Remark.}

The figures one and two illustrate functions $\underline{v}$ and $\bar{v}$ in the two differents cases: $p_{a}<M / 2$ and $p_{a} \geq M / 2$.

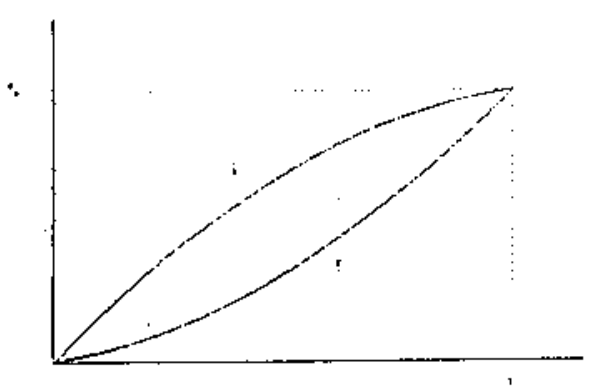

Fig. $1\left(p_{a}<M / 2\right)$

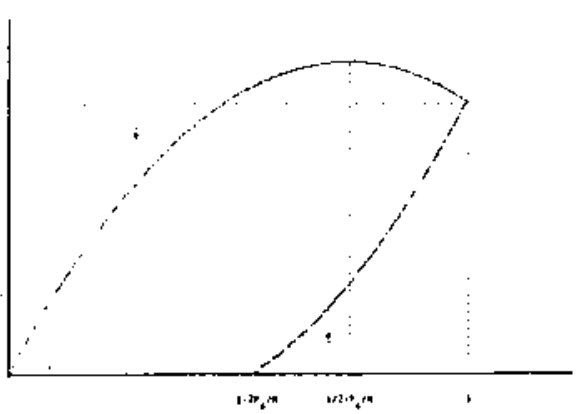

Fig. $2\left(p_{a} \geq M / 2\right)$

Figure 1 illustrate the region $(0,2 \pi) \times\left(0,1-2 p_{a} / M\right)$ where the free-boundary (when it exist) lies. The function $\bar{v}$ attain a maximum in $y=\frac{1}{2}+p_{a} / M \in(0,1)$; we shall prove later that, fixed $x, p(x, \cdot)$ is a non-decreasing monotone function up to this point.

Figure 2 corresponds to the case where there is not free-boundary; when $p_{a}>M / 2$ the solution is very close to the function $w(y)=p_{a} y$, which satisfies that $\operatorname{div}\left(h^{3} \nabla w\right)=0$, corresponding to the limit case when the eccentricity ratio $\alpha$ is equal to zero, and evidencing that this eccentricity is negligible when the pression on the supply line is very great.

\section{Behaviour of the free-boundary in the $y$-variable}

We consider in this section the case $p_{a}<M / 2$, denoting by $y_{m}$ the value $y_{m}=\frac{1}{2}+p_{a} / M$, where the function $\bar{v}$, defined by $(2.1)$, attain a maximum. Let $y_{0}=2 p_{a} / M$, and take $y_{1}$ any value in $\left(y_{m}, 1\right)$. Finally, let $\Omega_{1}=(0,2 \pi) \times\left(y_{0}, y_{1}\right)$, denoting by $\Gamma_{0}^{1}$ and $\Gamma_{1}^{1}$ the lower and upper boundarys of $\Omega_{1}$ respectively. (see Fig. 3).

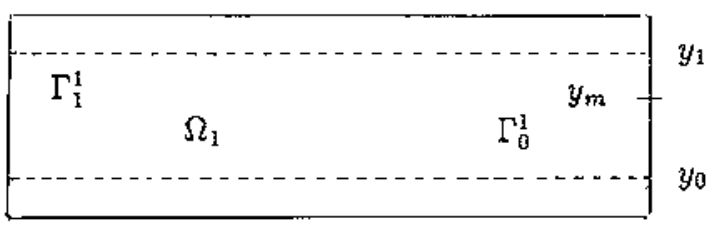

Fig. 3 
The equation $z=y_{1}-\frac{1}{\beta}\left(y-y_{1}\right)$ with $\beta=\frac{y_{1}-y_{0}}{1-y_{2}}$, transform the interval $\left[y_{0}, y_{1}\right]$ into $\left[y_{1}, 1\right]$. Making use of this transformation we can define a new function on $\Omega_{1}$, from the solution $p$ of Problem $(P)$, by means of

$$
\text { for }(x, y) \in \vec{\Omega}_{1} \quad \bar{p}(x, y)=\beta^{2} p(x, z) .
$$

We have:

\section{Theorem 3.1.}

$$
p(x, y) \leq \bar{p}(x, y) \quad \text { for any }(x, y) \in \Omega_{1} .
$$

Before to give the proof of Theorem 9.1 we shall first prove some previous results about $\bar{p}(x, y)$. We remark that the technics to prove this theorem are the same that the ones used to prove uniqueness. They are based on the construction of a class of test functions defined in a multidimensional domain. Sucht test functions appear in $[\mathrm{A}-\mathrm{C}],[\mathrm{C}-1]$ and $[\mathrm{C}-2]$.

\section{Proposition 3.2.}

If $(p, \gamma)$ is the solution of Problem $(P)$ and we define $\bar{\gamma}(x, y)=\gamma(x, z)$ for $(x, y) \in \Omega_{1}$, then the pair $(\bar{p}, \bar{\gamma})$ satisfies,

$$
\int_{\Omega_{1}} h^{3} \nabla \bar{p} \nabla \xi+\frac{1-\beta^{2}}{\beta^{2}} \int_{\Omega_{3}} h^{3} \bar{p}_{x} \xi_{x}=\int_{\Omega_{1}} h \bar{\gamma} \xi_{x}
$$

for any $\xi \in H^{1}\left(\Omega_{1}\right), 2 \pi x$-periodic and $\left.\xi\right|_{\Gamma_{0}^{3} \cup \Gamma_{1}^{1}}=0$

$$
H(\bar{p}) \leq \bar{\gamma} \leq 1 \quad \text { a.e. in } \Omega
$$

Moreover

$$
\left.p\right|_{\Gamma_{0}^{1} \cup \Gamma_{1}^{1}} \leq\left.\bar{p}\right|_{\Gamma_{0}^{2} \cup \Gamma_{1}^{1}}
$$

Proof: Let $\bar{\xi}(x, z)=\xi(x, y), J=\left(\begin{array}{cc}1 & 0 \\ 0 & -1 / \beta\end{array}\right)$ the matrix for derivatives of $(x, z)$ with rapport to $(x, y)$, and $\Omega_{2}=(0,2 \pi) \times\left(y_{1}, 1\right)$ with lower and upper boundarys $\Gamma_{0}^{2}$ and $\Gamma_{1}^{2}$ respectively $\left(\Gamma_{0}^{2}=\Gamma_{1}^{1}\right.$, and $\left.\Gamma_{1}^{2}=\Gamma_{1}\right)$. We get:

$$
\begin{aligned}
& \int_{\Omega_{1}} h^{3} \nabla \bar{p} \nabla \xi=\int_{\Omega_{1}} h^{3} \nabla_{x, y} \beta^{2} p(x, z) \nabla_{x, y} \xi(x, y) d x d y= \\
& =\int_{\Omega_{2}} h^{3}\left(\nabla_{x, z} \beta^{2} p(x, z) J\right) \cdot\left(\nabla_{x, z} \bar{\xi}(x, z) J\right) \beta d x d z= \\
& =\beta \int_{\Omega_{2}} h^{3}\left\{\beta^{2} p_{x} \bar{\xi}_{x}+p_{z} \bar{\xi}_{z}\right\} d x d z= \\
& =\beta \int_{\Omega_{2}} h^{3} \nabla_{x, z} p \nabla_{x, z} \bar{\xi} d x d z+\beta\left(\beta^{2}-1\right) \int_{\Omega_{z}} h^{3} p_{x} \bar{\xi}_{x} d x d z= \\
& =\beta \int_{\Omega_{2}} h \gamma \bar{\xi}_{x} d x d z+\beta\left(\beta^{2}-1\right) \int_{\Omega_{2}} h^{3} p_{x} \bar{\xi}_{x} d x d z
\end{aligned}
$$


since $\bar{\xi} \in H^{1}\left(\Omega_{2}\right)$, is $2 \pi x$-periodic and $\left.\bar{\xi}\right|_{\Gamma_{0}^{2} \cup \Gamma_{1}^{2}}=0$.

Now, coming back to the $y$-variable in $\Omega_{1}$, we conclude

$$
\int_{\Omega_{1}} h^{3} \nabla \bar{p} \nabla \xi+\frac{1-\beta^{2}}{\beta^{2}} \int_{\Omega_{1}} h^{3} \bar{p}_{x} \xi_{x}=\int_{\Omega_{1}} h \bar{\gamma} \xi_{x}
$$

and $H(\bar{p})=H\left(\beta^{2} p(x, z)\right) \leq \gamma(x, z)=\bar{\gamma}(x, y) \leq 1$ a.e. in $\Omega_{1}$.

Moreover $p \leq \bar{p}$ on $\Gamma_{1}^{3}$, because $y=z$ and $\beta^{2}>I$, and $p \leq p_{a} \leq \beta^{2} p_{a}=\bar{p}$ on $\Gamma_{0}^{\mathrm{I}}$.

We shall distinguish the $x$-variable for $p$ and $\bar{p}$, using the variables $\left(x_{1}, y\right) \in$ $\Omega_{1}$ for $(p, \gamma)$ and $\left(x_{2}, y\right) \in \Omega_{1}$ for $(\vec{p}, \bar{\gamma})$; we set $Q=(0,2 \pi) \times(0,2 \pi) \times\left(y_{0}, y_{1}\right)$, and let us consider $\xi(r)$ and $\rho(r)$, real functions such that:

$$
\begin{gathered}
\xi(r) \in C_{0}^{\infty}\left(y_{0}, y_{1}\right), \quad \xi \geq 0 . \\
\rho(r) \in C_{0}^{\infty}(R), \quad \rho \geq 0, \operatorname{supp} \rho=[-1,1] \\
\rho \text { is a pair function. }
\end{gathered}
$$

For small $\varepsilon>0$ we define $\rho_{\varepsilon}(r)=(1 / \varepsilon) \rho(r / \varepsilon)$, and finally for $\left(x_{1}, x_{2}, y\right) \in \vec{Q}$ let $F\left(x_{1}, x_{2}, y\right)$ be defined by

$$
F\left(x_{1}, x_{2}, y\right)=\xi(y) \rho_{\varepsilon}\left(\frac{x_{1}-x_{2}}{2}\right)
$$

This function, is identically zero when $\left|x_{1}-x_{2}\right| \geq 2 \varepsilon$ and, since $\rho_{\varepsilon}$ is a pair function, it can be redefined when $\left(x_{1}, x_{2}\right) \in T_{1} \cup T_{2}=\left\{\left(x_{1}, x_{2}\right) \in\right.$ $\left.[0,2 \pi] \times[0,2 \pi]:\left|x_{1}-x_{2}\right| \geq 2 \pi-2 \varepsilon\right\}$, by making

$$
\rho_{\varepsilon}\left(\frac{x_{1}-x_{2}}{2}\right)=\rho_{\varepsilon}\left(\frac{\left|x_{1}-x_{2}\right|-2 \pi}{2}\right)
$$

So we obtain a $2 \Gamma$-periodic function in the independents variables $x_{1}$ and $x_{2}$ (see Fig. 4). Moreover $F\left(\cdot, x_{2}, \cdot\right), F\left(x_{1}, \cdot, \cdot\right) \in H^{1}\left(\Omega_{1}\right)$ and $F\left(x_{1}, x_{2}, y_{0}\right)=$ $F\left(x_{1}, x_{2}, y_{1}\right)=0$.

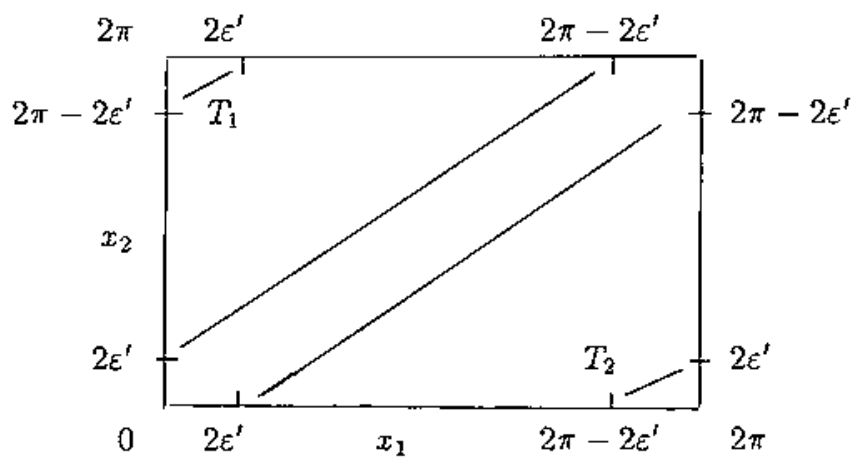

Fig. 4 
Now, let us consider a new parameter $\delta>0$, and define

$$
\eta\left(x_{1}, x_{2}, y\right)=\operatorname{Min}\left[\frac{\left(p\left(x_{1}, y\right)-\bar{p}\left(x_{2}, y\right)\right)^{+}}{\delta}, F\right]
$$

Using this function and denoting by $\nabla_{1}$ and $\nabla_{2}$ the gradient operator for $\left(x_{1}, y\right)$ and $\left(x_{2}, y\right)$ respectively, we have:

\section{Proposition 3.3 .}

$$
\int_{Q}\left\{\left(h^{3}\left(x_{1}\right) p_{x_{1}}-h^{3}\left(x_{2}\right) \bar{p}_{x_{2}}\right)\left(\eta_{x_{1}}+\eta_{x_{2}}\right)+\left(h^{3}\left(x_{1}\right) p_{y}-h^{3}\left(x_{2}\right) \bar{p}_{y}\right) \eta_{y}\right\}+
$$

$$
+\frac{\beta^{2}-1}{\beta^{2}} \int_{Q} h^{3}\left(x_{2}\right) \ddot{p}_{x_{2}}\left(\eta_{x_{2}}+\eta_{x_{2}}\right)=\int_{Q}\left(h\left(x_{1}\right)-h\left(x_{2}\right) \bar{\gamma}\right)\left(\eta_{x_{1}}+\eta_{x_{2}}\right)
$$

Proof: For each $x_{2} \in(0,2 \pi)$, we have

$$
\begin{aligned}
& \int_{\Omega_{1}} h^{3}\left(x_{1}\right) \nabla_{1} p \nabla_{1} \eta d x_{1} d y=\int_{\Omega_{1}} h\left(x_{1}\right) \eta_{x_{1}} d x_{1} d y= \\
& =\int_{\Omega_{1}}\left(h\left(x_{1}\right)-h\left(x_{2}\right) \bar{\gamma}\right) \eta_{x_{1}} d x_{1} d y
\end{aligned}
$$

since $\gamma_{1} \equiv 1$ on $\operatorname{supp} \eta\left(\cdot, x_{2}, \cdot\right)$, and $\int_{\Omega_{2}} h\left(x_{2}\right) \bar{\gamma} \eta_{x_{1}}=0$, from the periodicity of $\eta$.

By integrating the above equality in the $x_{2}$-variable, we get:

$$
\int_{Q} h^{3}\left(x_{1}\right) \nabla_{1} p \nabla_{1} \eta=\int_{Q}\left(h\left(x_{1}\right)-h\left(x_{2}\right) \bar{\gamma}\right) \eta_{x_{1}}
$$

and, analogously for $\bar{p}$ :

$$
\begin{aligned}
& \int_{Q} h^{3}\left(x_{2}\right) \nabla_{2} \bar{p} \nabla_{2} \eta+\frac{1-\beta^{2}}{\beta^{2}} \int_{Q} h^{3}\left(x_{2}\right) \bar{p}_{x_{2}} \eta_{x_{2}}= \\
& =\int_{Q}\left(h\left(x_{2}\right) \bar{\gamma}-h\left(x_{1}\right)\right) \eta_{x_{2}}
\end{aligned}
$$

Substracting the above equalities, we get:

$$
\begin{aligned}
& \int_{Q}\left(h^{3}\left(x_{1}\right) \nabla_{1} p \nabla_{1} \eta-h^{3}\left(x_{2}\right) \nabla_{2} \bar{p} \nabla_{2} \eta\right)+\frac{\beta^{2}-1}{\beta^{2}} \int_{Q} h^{3}\left(x_{2}\right) \vec{p}_{x_{2}} \eta_{x_{2}}= \\
& =\int_{Q}\left(h\left(x_{1}\right)-h\left(x_{2}\right) \bar{\gamma}\right)\left(\eta_{x_{1}}+\eta_{x_{2}}\right)
\end{aligned}
$$


Moreover

$$
\int_{Q} h^{3}\left(x_{2}\right) \bar{p}_{x_{2}} \eta_{x_{1}}=\int_{Q} h^{3}\left(x_{1}\right) p_{x_{1}} \eta_{x_{2}}=0
$$

and introducing this terms in (3.5), we conclude (3.4).

Now, we go to consider the new variables (see $[\mathbf{A}-\mathbf{C}],[\mathrm{C}-1]$ ):

$$
t=\frac{x_{1}+x_{2}}{2}, \quad z=\frac{x_{1}-x_{2}}{2},
$$

getting for the function $\eta$ :

$$
\eta(t+z, t-z, y)=\operatorname{Min}\left[\frac{(p(t+z, y)-\bar{p}(t-z, y))^{+}}{\delta}, \xi(y) \rho_{\varepsilon}(z)\right]
$$

and, for derivatives:

$$
\begin{aligned}
& \phi_{x_{1}}=\frac{1}{2}\left(\phi_{t}+\phi_{z}\right), \\
& \phi_{x_{2}}=\frac{1}{2}\left(\phi_{t}-\phi_{z}\right), \quad \text { for any } \phi=\phi\left(x_{1}, x_{2}, y\right) \\
& \phi_{x_{1}}+\phi_{x_{2}}=\phi_{t}
\end{aligned}
$$

what, in the particular case of $p=p\left(x_{1}, y\right)$ and $\bar{p}=\bar{p}\left(x_{2}, y\right)$, being $p_{t}(t+z, y)=$ $p_{z}(t+z, y)$ and $\bar{p}_{\ell}(t-z, y)=-\bar{p}_{z}(t-z, y)$, gives:

$$
\begin{aligned}
& p_{x_{1}}\left(x_{1}, y\right)=p_{t}(t+z, y) \\
& \bar{p}_{x_{2}}\left(x_{2}, y\right)=\bar{p}_{t}(t-z, y) .
\end{aligned}
$$

In the new variables, the equation (3.4) becomes:

$$
\begin{aligned}
& \int_{Q_{t z}}\left(h^{3}(t+z) \nabla_{t y} p(t+z, y)-h^{3}(t-z) \nabla_{t y} \bar{p}(t-z, y)\right) \nabla_{t y} \eta+ \\
& +\frac{\beta^{2}-1}{\beta^{2}} \int_{Q_{t z}} h^{3}(t-z) \bar{p}_{t} \eta_{t}=\int_{Q_{t z}}(h(t+z)-h(t-z) \bar{\gamma}) \eta_{t},
\end{aligned}
$$

where we omite the constant due to the coordinates transformation, and denote by $Q_{t z}$ the new domain.

If we consider the sets,

$$
A_{\varepsilon}^{\delta}=\left[\left(p_{1}-p_{2}\right)^{+}>\delta \xi \rho_{\varepsilon}\right] \quad B_{\varepsilon}^{\delta}=\left[0<p_{1}-p_{2} \leq \delta \xi \rho_{\varepsilon}\right]
$$


(in $Q$ or $Q_{t z}$ ) and denote:

$$
\begin{aligned}
I_{1} & =\int_{A_{c}^{\delta}}\left(h^{3}(t+z) \nabla_{t y} p(t+z, y)-h^{3}(t-z) \nabla_{t y} \bar{p}(t-z, y)\right) \nabla_{t y}\left(\xi(y) \rho_{\varepsilon}(z)\right)= \\
& =\int_{A_{c}^{\delta}}\left(h^{3}(t+z) p_{y}(t+z, y)-h^{3}(t-z) \bar{p}_{y}(t-z, y)\right) \xi^{\prime}(y) \rho_{\varepsilon}(z) \\
I_{2} & =\int_{B_{\varepsilon}^{\delta}}\left(h^{3}(t+z) \nabla_{t y} p(t+z, y)-h^{3}(t-z) \nabla_{t y} \bar{p}(t-z, y)\right) \nabla_{t y} \frac{p-\bar{p}}{\delta} \\
I_{3} & =\frac{\beta^{2}-1}{\beta^{2}} \int_{B_{\varepsilon}^{\delta}} h^{3}(t-z) \bar{p}_{t} \frac{(p-\bar{p})_{t}}{\delta} \\
I_{4} & =\int_{B_{\varepsilon}^{\delta}}(h(t+z)-h(t-z) \bar{\gamma}) \frac{(p-\bar{p})_{t}}{\delta}
\end{aligned}
$$

we can write (3.6) in the form:

$$
I_{1}+I_{2}+I_{3}=I_{4} .
$$

For $I_{4}$ we have:

Lemma 3.4. ([A])

$$
\lim _{\varepsilon \rightarrow 0}\left[\lim _{\delta \rightarrow 0} I_{4}\right]=0
$$

Let us prove now, the following:

\section{Lemma 3.5.}

$$
\lim _{\varepsilon \rightarrow 0}\left[\lim _{\delta \rightarrow 0} I_{1}\right] \leq 0
$$

Proof:

$$
\begin{aligned}
& I_{2}+I_{3}=\int_{B_{c}^{\delta}}\left(h^{3}(t+z)\left|\nabla_{t y} \frac{p}{\delta}\right|^{2}+h^{3}(t-z)\left|\nabla_{t y} \frac{\bar{p}}{\delta}\right|^{2}\right)- \\
& -\int_{B_{\varepsilon}^{\delta}}\left(h^{3}(t+z) \nabla_{t y} p \nabla_{t y} \frac{\bar{p}}{\delta}+h^{3}(t-z) \nabla_{t y} \bar{p} \nabla_{t y} \frac{p}{\delta}\right)+ \\
& +\frac{\beta^{2}-1}{\beta^{2}} \int_{B_{\varepsilon}^{\delta}} h^{3}(t-z) \bar{p}_{t}\left(\frac{p}{\delta}\right)_{t}-\frac{\beta^{2}-1}{\beta^{2}} \int_{B_{\varepsilon}^{\delta}} h^{3}(t-z)\left(\frac{\bar{p}_{t}}{\delta}\right)^{2}
\end{aligned}
$$

denoted by $J_{1}-J_{2}+J_{3}-J_{4}$; with the following balance:

$$
J_{1}-J_{4} \geq 0 \text { because } 0<\frac{\beta^{2}-1}{\beta^{2}}<1 \text {. }
$$

$\left|J_{3}\right| \leq\left|J_{2}\right|$ and $J_{2}$ can be decomposed in two integrals having both of them Iimit equal to zero, when we pass to the limit first as $\delta \rightarrow 0$ and later as $\varepsilon \rightarrow 0$. (see $[\mathbf{A}],[\mathbf{A}-\mathbf{C}])$. 
From Lemma 3.4 and (3.7) we conclude (3.8).

Proof of Theorem 3.1: By Lebesgue Theorem,

$$
\begin{aligned}
& \lim _{\delta \rightarrow 0} I_{1}=\int_{Q_{t z}}\left(h^{3}(t+z) \frac{\partial}{\partial y} p-h^{3}(t-z) \frac{\partial}{\partial y} \bar{p}\right) \chi([p>\bar{p}]) \xi^{\prime}(y) \rho_{\varepsilon}(z)= \\
& =\int_{Q_{t z}} h^{3}(t+z) \frac{\partial}{\partial y}(p-\bar{p}) \chi([p>\bar{p}]) \xi^{\prime}(y) \rho_{e}(z)+ \\
& +\int_{Q_{i z}}\left(h^{3}(t+z)-h^{3}(t-z)\right) \frac{\partial}{\partial y} \bar{p} \chi([p>\bar{p}]) \xi^{\prime}(y) \rho_{\varepsilon}(z)
\end{aligned}
$$

denoted by $I_{1}^{1}$ and $I_{1}^{2}$ respectily.

$r_{1}^{2}$ satisfies

$$
\begin{aligned}
& \left|I_{1}^{2}\right| \leq C \int_{Q_{t z}}\left|h^{3}(t+z)-h^{3}(t-z) \| \frac{\partial}{\partial y} \vec{p}\right| \rho_{\varepsilon}(z) \leq \\
& \leq C\left\|\frac{\partial}{\partial y} \bar{p}\right\|_{L^{2}\left(Q_{t z}\right)}\left\{\int_{Q_{t z}}\left|h^{3}(t+z)-h^{3}(t-z)\right|^{2}\left|\rho_{\varepsilon}(z)\right|^{2}\right\}^{1 / 2} \leq C^{\prime} \sqrt{\varepsilon}
\end{aligned}
$$

because $h^{3}$ is Lipschitz continuous and the measure of $\operatorname{supp} \rho_{\varepsilon}(z)$ is $4 \pi \varepsilon$, and then

$$
\int_{Q_{t z}}\left|h^{3}(t+z)-h^{3}(t-z)\right|^{2}\left|\rho_{\varepsilon}(z)\right|^{2} \leq \operatorname{cte} \int_{Q_{z z}}|z|^{2} \frac{1}{\varepsilon^{2}}\left(\rho_{\varepsilon}(z / \varepsilon)\right)^{2} \leq \text { cte } \varepsilon
$$

From (3.8) we have:

$$
\begin{aligned}
0 & \geq \lim _{\varepsilon \rightarrow 0}\left[\lim _{\delta \rightarrow 0} I_{\mathrm{I}}\right]=\lim _{\varepsilon \rightarrow 0} I_{1}^{1}+\lim _{\varepsilon \rightarrow 0} I_{1}^{2}= \\
& =\lim _{\varepsilon \rightarrow 0} \int_{Q_{t z}} h^{3}(t+z) \frac{\partial}{\partial y}\left[(p-\bar{p})^{+}\right] \xi^{\prime}(y) \rho_{\varepsilon}(z)= \\
& =-\lim _{\varepsilon \rightarrow 0} \int_{Q_{t z}} h^{3}(t+z)(p-\bar{p})^{+} \xi^{\prime \prime}(y) \rho_{\varepsilon}(z)
\end{aligned}
$$

but, by a classical argument (see [A]) we can elimine $\varepsilon$ and the $z$-variable, concluding:

$$
\int_{\Omega_{1}} h^{3}(t)(p(t, y)-\bar{p}(t, y))^{+} \xi^{\prime \prime}(y) d t d y \geq 0
$$

Now, setting

$$
T(y)=\int_{0}^{2 \pi} h^{3}(t)(p-\tilde{p})^{+} d t
$$


(3.9) is equivalent to:

$$
\left\langle\frac{d^{2} T}{d y^{2}}, \xi\right\rangle_{\mathcal{D}^{\prime}\left(y_{0}, y_{1}\right) \times \mathcal{D}\left(y_{0}+y_{1}\right)} \geq 0
$$

and we have that the distribution $T$ satisfies:

$$
\begin{aligned}
& \frac{d^{2} T}{d t_{2}^{2}} \geq 0 . \\
& T(0)=T(1)=0 \quad \text { due to }(3.3) .
\end{aligned}
$$

Hence, by the maximum principle, we conclude

$$
\int_{0}^{2 \Pi} h^{3}(t)(p-\bar{p})^{+} d t \leq 0
$$

and then

$$
p \leq \bar{p} \quad \text { in } \Omega_{1}
$$

That is,

$$
p(x, y) \leq \beta^{2} p(x, \bar{y})=\beta^{2} p\left(x, y_{1}-\frac{1}{\beta}\left(y-y_{1}\right)\right)
$$

and the proof ends.

When $y_{1} \leq y_{m}$ (the point of a maximum for $\bar{v}(y)$ ), we can obtain the same result with $\beta=1$. We introduce two cases:

If $1 / 2<y_{1} \leq y_{m}$, we make $y_{0}=2 y_{1}-1, \Omega_{1}=(0,2 \pi) \times\left(y_{0}, y_{1}\right)$, $\Omega_{2}=(0,2 \pi) \times\left(y_{1}, 1\right)$ and $z=2 y_{1}-y$.

If $y_{1} \leq 1 / 2$, we make $y_{0}=0, \quad \Omega_{1}=(0,2 \pi) \times\left(0, y_{1}\right)$,

$\Omega_{2}=(0,2 \pi) \times\left(y_{1}, 2 y_{1}\right)$ and $z=2 y_{1}-y$ (see Fig. 5 and 6$)$.

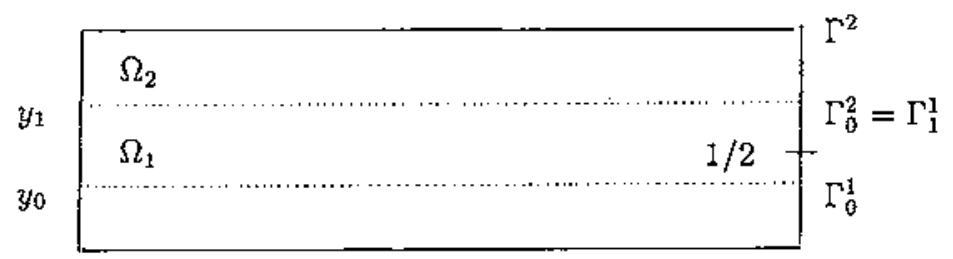

Fig. 5 


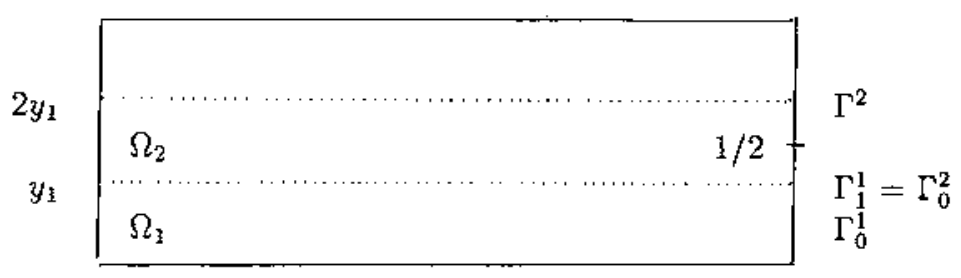

Fig. 6

For both cases $\beta=1,\left.p\right|_{\Gamma_{0}^{1}} \leq\left. p\right|_{\Gamma_{1}^{2}}$ and we can conclude as in Theorem 3.1:

Corollary 3.6.

If $(p, \gamma)$ is the solution of Problem $(P)$, then $p(x, \cdot)$ is a monotone incresing function on $\left[0, y_{m}\right]$.

Proof: Let $y^{1}, y^{2} \in\left[0, y_{m}\right]$ and such that $y^{1}<y^{2}$; taking $y_{1}=\frac{y^{2}+y^{2}}{2}$, we have $y^{2}=2 y_{1}-y^{1}$, and applying Theorem 3.1 , we conclude

$$
p\left(x, y^{1}\right) \leq p\left(x, y^{2}\right) \text {. }
$$

Corollary 3.7.

Let $(x, z)$ be such that $p(x, z)=0$; then $p(x, y)=0$ for any $y \in[0, z]$.

Proof: By the above Corollary we must only to prove that $p(x, y)=0$ in $\left[y_{m}, z\right]$ when $z>y_{m}$.

For $y \in\left[y_{m}, z\right)$, we take $y_{1} \in(y, z)$ such that $y-y_{1}=-\frac{y_{1}-y_{0}}{1-y_{2}}\left(z-y_{1}\right)$ (see Fig. 7), which is equivalent to $z=y_{1}-\frac{1}{\beta}\left(y-y_{1}\right)$ with $\beta=\frac{y_{1}-y_{0}}{1-y_{1}}>1$.

Applying Theorem 3.1 , we conclude:

$p(x, y) \leq \beta^{2} p(x, z)=0$ for any $y \in\left[y_{m}, z\right)$, and hence $p(x, y)=0$ for any $y \in[0, z]$.

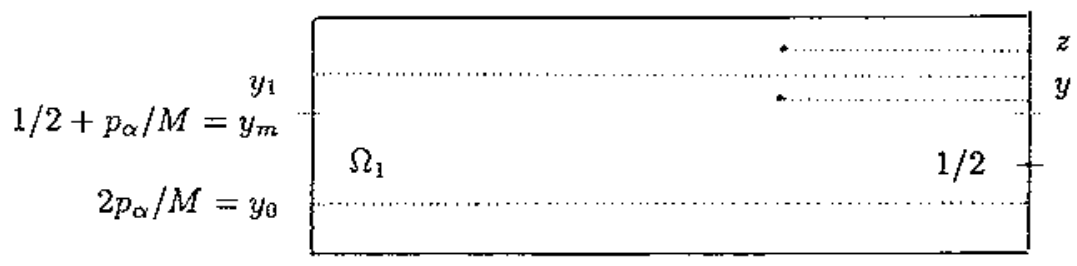

Fig. 7 
Remark. Corollary 3.7 states that the free-boundary does not have horizontal oscillations.

\section{Behaviour of $\gamma$ in the $x$-variable}

We go to study some properties of $\gamma$ with geometrical consequences on the free-boundary, when $x \in(0, \pi)$.

Theorem 4.1.

Let $(p, \gamma)$ be the solution of Problem $(P)$, and let $\chi$ be the characteristic function of the set $[p>0]$; then,

$$
(h \gamma)_{x}-h^{\prime} \chi \geq 0 \quad \text { in } \mathcal{D}^{\prime}(\Omega)
$$

Proof: Let $\phi \in \mathcal{D}(\Omega)$ with $\phi \geq 0$, and for $\varepsilon>0$ let us consider the test function $\xi=\min (\varepsilon \phi, p)$; we have:

$$
\int_{\Omega} h^{3} \nabla p \nabla \xi=\int_{[p<\varepsilon \phi]} h^{3}|\nabla p|^{2}+\varepsilon \int_{[p \geq \varepsilon \phi]} h^{3} \nabla p \nabla \phi=\int_{\Omega} h \xi_{x}=-\int_{\Omega} h^{\prime} \xi
$$

since $\gamma=1$ on the support of $\xi$. Then

$$
\int_{[p \geq \epsilon \phi]} h^{3} \nabla p \nabla \phi+\int_{\Omega} h^{\prime} \min (\phi, p / \varepsilon)=-1 / \varepsilon \int_{[p<\epsilon \phi]} h^{3}|\nabla p|^{2} \leq 0
$$

letting $\varepsilon \rightarrow 0$ and using the Lebesgue Theorem, we obtain:

$$
\int_{\Omega} h^{3} \nabla p \nabla \phi+\int_{\Omega} h^{\prime} \chi \phi \leq 0
$$

but

$$
\int_{\Omega} h^{3} \nabla p \nabla \phi=\int_{\Omega} h \gamma \phi_{x}
$$

concluding that

$$
\int_{\Omega} h \gamma \phi_{x}+\int_{\Omega} h^{\prime} \chi \phi \leq 0 \quad \forall \phi \in \mathcal{D}^{+}(\Omega)
$$

which equivales to

$$
\left\langle h^{\prime} \chi-(h \gamma)_{x}, \phi\right\rangle_{\mathcal{D}^{\prime}(\Omega) \times \mathcal{D}(\Omega)} \leq 0 \quad \forall \phi \in \mathcal{D}^{+}(\Omega) .
$$

and, hence

$$
h^{\prime} \chi-(h \gamma)_{x} \leq 0 \quad \text { in } \mathcal{D}^{\prime}(\Omega)
$$


Corollary 4.2.

$$
\begin{array}{lr}
\gamma_{x} \geq 0 & \text { in } \mathcal{D}^{\prime}((0, \pi) \times(0,1)) . \\
(h \gamma)_{x} \geq 0 & \text { in } \mathcal{D}^{\prime}((\pi, 2 \pi) \times(0,1)) .
\end{array}
$$

Proof: As $h^{\prime}>0$ in $(\pi, 2 \pi)$ and from (4.1) we deduce that

$$
(h \gamma)_{x} \geq h^{\prime} \chi \geq 0 \quad \text { in } \mathcal{D}^{\prime}((\pi, 2 \pi) \times(0,1)) .
$$

In $(0, \pi)$ :

$$
\begin{aligned}
& h^{\prime} \chi-(h \gamma)_{x}=h^{\prime} \chi-h^{\prime} \gamma-h \gamma_{x}=h^{\prime}(\chi-\gamma)-h \gamma_{x} \leq 0 \\
& h^{\prime}<0 \\
& \chi-\gamma \leq 0
\end{aligned}
$$

so that,

$$
\gamma_{x} \geq \frac{h^{\prime}(x-\gamma)}{h} \geq 0 \quad \text { in } \mathcal{D}^{\prime}((0, \pi) \times(0,1))
$$

\section{Corollary 4.3.}

If $p\left(x_{0}, y_{0}\right)>0$ for some $x_{0}<\pi$, then there exists $\varepsilon>0$ such that $p>0$ on the set $C_{\varepsilon}=\left(x_{0}-\varepsilon, \pi\right) \times\left(y_{0}-\varepsilon, y_{0}+\varepsilon\right)$.

Proof: From the continuity of $p$, there exist $Q_{\varepsilon}=\left(x_{0}-\varepsilon, x_{0}+\varepsilon\right) \times\left(y_{0}-\right.$ $\varepsilon, y_{0}+\varepsilon$ ) such that $p>0$ in $Q_{\varepsilon}$ (sce Fig. 8) and $\gamma=1$ a.e. in $Q_{\varepsilon}$. Like $\gamma_{r} \geq 0$ we get $\gamma=1$ a.e. in $C_{\varepsilon}$.

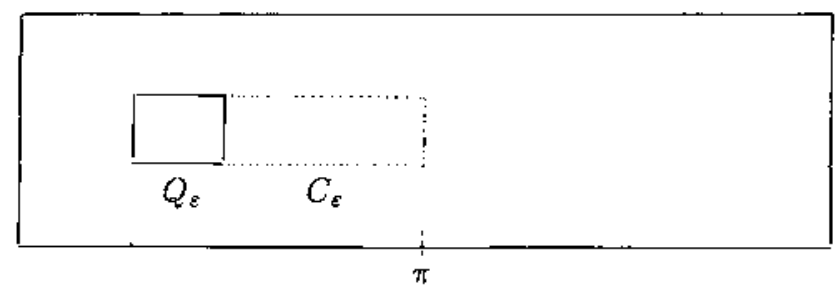

Fig. 8

Now, for $\phi \in C_{0}^{\infty}\left(C_{\varepsilon}\right)$ we have

$$
\int_{C_{\varepsilon}} h^{3} \nabla p \nabla \phi=\int_{C_{\varepsilon}} h \phi_{x}
$$

and, hence

$$
\operatorname{div} h^{3} \nabla p=h^{\prime}<0 \quad \text { in } \mathcal{D}^{\prime}\left(C_{\varepsilon}\right)
$$


Using the strong minimum principle, $p$ can not attain the minimum value zero in $C_{\varepsilon}$ and hence

$$
p>0 \quad \text { in } C_{\varepsilon} .
$$

Remark. As a consequence of this Corollary the free-boundary can not have vertical oscillations in the interval $(0, \pi)$.

Taking account the Corollary 3.7 , we conclude that the free-boundary is a monotone decreasing graph $-y=\Gamma(x)$-in the interval $(0, \pi)$ (see Fig. 9).

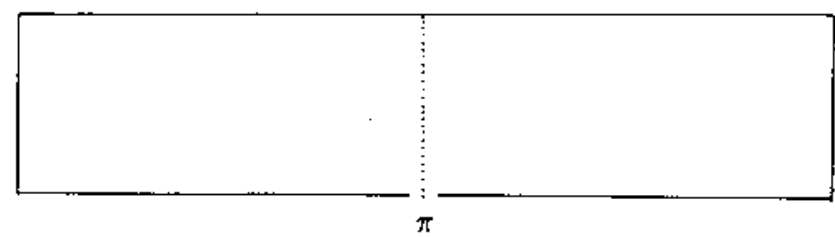

Fig. 9

Theorem 4.4.

If $(p, \gamma)$ is the solution of Problem $(P)$, then $p$ satisfies:

$$
\int_{0}^{2 \pi} h^{3}(x) p(x, y) d x=p_{a} y \int_{0}^{2 \pi} h^{3}(x) d x
$$

Proof: For $\phi(y) \in C_{0}^{\infty}(0,1)$ we have

$$
\int_{\Omega} h^{3} \nabla p \nabla \phi=\int_{\Omega} h^{3} p_{y} \phi^{\prime}=0
$$

Integrating by parts and introducing the function

we have

$$
F(y)=\int_{0}^{2 \pi} h^{3}(x) p(x, y) d x
$$

$$
\left\langle\frac{d^{2} F}{d y^{2}}, \phi\right\rangle_{\mathcal{D}^{\prime}(0,1) \times \mathcal{D}(0,1)}=0
$$

and hence

$$
\frac{d^{2} F}{d y^{2}}=0 \quad \text { in } \mathcal{D}^{\prime}(0,1)
$$

but, $F(0)=0$ and $F(1)=p_{a} \int_{0}^{2 \pi} h^{3}$, so we conclude

$$
F(y)=p_{a} y \int_{0}^{2 \pi} h^{3}(x) d x .
$$

Corollary 4.5.

Given $y \in(0,1)$ there exist a region of positive measure in $(0,2 \pi)$ where $p>0$. 


\section{References}

[A] J.S. A LVAREZ, Problemas de Frontera Libre en Teoría de Lubrificación, Tesis Doctoral (1986).

[A-C] J.S. Alvarez, J. CARRILlo, A Free-Boundary Problem in Theory of Lubrication, to appear in Communications in Partial Differential Equations.

[B-Ch] G. BAYADA, M. Chambat, Existence and Uniqueness for a Lubrication Problem with Non-Regular Conditions on the Free-Boundary, B.U.M.I. 6, 3-B (1984).

[B-K-S] H. BrézIS, D. KInderlHerer, D. StampaCchia, Sur une nouvelle formulation du probléme de l'écoulement à travers une digue, C.R.A.S. de Paris 287 Série A, 711-714.

[C-1] J. CARRILLO, On the uniqueness of the solution of a class of elliptic equations with nonlinear convection, Contributions to Nonlinear Partial Differential Equations II. Pitman Research Notes in Mathematics Series.

[C-2] J. CARRILLO, Unicité des solutions du type Krouskov pour des problémes elliptiques avec des termes de transport non linéaires, C.R.A.S. 909 Série I, no. 5 (1986).

[D-T] D. DowsOn, C.M. TAYLOR, Cavitation in Bearings, Annual Review of Fluids Mechanics 11 (1979).

[F] L. FLOBERG, Cavitation in Lubricating oil Films.

Escuela Técnica Superior de Arquitectura

Universidad Politécnica de Madrid

Madrid-28040

SPAIN

Rebut el 22 de Desembre de 1988 